\title{
Gender, Age and Social Stigma: The Coping strategy Choice on Mental Health and Attitudes Towards Help-seeking
}

\author{
Jiani Zhang* \\ International Department of Chengdu No.7 High School, Chengdu, Sichuan610041, China, \\ nny2004415@gmail.com
}

\begin{abstract}
The tendency of many men not to seek help and dilute their physical and mental health symptoms has become a common public image of men. Admitting a problem and asking for help may be seen as a sign of weakness for men, so they associate seeking psychological or emotional help with shame or weakness. This paper focuses on the significance of gender to help-seeking. The first objective of this study is to explore the differences in the choice with mental health problems between different genders. And another purpose of this study was to explore gender differences in attitudes towards seeking professional psychological help. This work analyzed the self-questionnaire about a hundred and twenty-seven participants of both sexes ranging from ages 15-50. Participants responded to 6 questions, including 3 basic pieces of information and 3 questions assessing their attitudes toward help-seeking. To investigate the self-questionnaire statistically, this work performed a Chi-Square Test of Independence in Excel to predict the association between gender and coping strategies when encountering psychological barriers, and between gender and people's attitudes towards help-seeking. Since the p-value in each part is not less than 0.05 , the results cannot conclude that those associations are accurate. Although this work does not have enough solid data to support our two hypotheses, previous studies have confirmed the correlation between social stigma and gender, and we will further study the impact of social stigma on gender mental health, especially on help-seeking.
\end{abstract}

Keywords: gender; help-seeking; men; masculinity; social sigma; self-regulation

\section{INTRODUCTION}

\subsection{Background}

Gender differences have been a major focus and an essential element for researchers studying the behavior, choice, and mental health of people of both sexes. With the rise of the feminist movement in the past two years, more and more people are aware of the social and individual oppression caused by gender prejudice, and the "Equal rights" movement has been promoted worldwide. Gender stereotypes conventionalize people like a heavy shackle for both men and women. For men, this stigma undoubtedly limits their emotional reactions and has a profound impact on their mental health.

Jules Angst and Celile Ernst, who studied suicide prevention, concluded that "Women seek help, men die ". And previous research has established that women suffer from major depression at a higher rate than men [1], about twice as often. However, in all groups, suicide rates are higher for men than for women[2], about two to six times as likely. And previous research has established that the suicide rate of men in the UK was three times higher than that of women in 2019.

\subsection{The Significance of the Research}

An indispensable factor in this situation is social stigma. The effects of sexism mean that men may be less likely than women to discuss or seek support for suicidal thoughts or behavior. According to Mental Health statistics, 15 percent of women are willing to seek treatment for a health problem, compared with 9 percent of men.[3] Men who have experienced the negative effects of socialized gender roles show less positive attitudes and reluctance to seek counseling.[4]

Society imperceptibly formulates many labels for people, potentially regulating people's behavior and 
mentality. Women are expected to be gentle, who ought to be protected, while men are expected to be strong, self-reliant, and confident. Like toxic masculinity has gone mainstream in the past few years. It, as defined by the Oxford Dictionary, refers to the harmful belief that men should behave according to standards set by society, as well as emotional limits on what men should or should not have. Because suppressing emotional expression affects the perception of depressive symptoms, traditional male social norms make it harder for men to seek help.[5] In traditional parenting, men are often encouraged to develop strong, independent traits, which may make it difficult for them to form close and deep relationships with others. While women can choose to express their vulnerability and seek help, gender stereotypes of men as toxic masculinity do not allow for failure, which can lead to men being reluctant to show their "wounds" and limit them to seek help from their relatives, friends, or professional doctors. Expressing emotional weakness makes them feel unmanly and this is a barrier for males to accurately identify and treat psychological disorders.[6]

\subsection{The Purpose of The Research}

A study in the British Medical Journal discovered that the rates of general primary care consultation in men were $32 \%$ lower than in women.[7] The central issue is what causes the differences in the way men and women deal with mental problems. In this study, the work tended to discover the connection between gender and people's choice to deal with mental health problems and the different attitudes towards help-seeking of people in both sexes.

\section{METHODOLOGY}

\subsection{Assumptions}

In the work, 2 assumptions were formulated. The first assumption is that men are significantly less likely to seek help than women, and the second assumption is that men tend to hold more repellent attitudes towards help-seeking compared to women.
There were 127 participants in this sample. All the participants of both sexes range from ages 15-50 including 76 females, mainly from Chengdu and Shanghai. They were instructed to complete an online questionnaire consisting of 6 questions asking about their mental well-being and attitudes towards help-seeking. The questionnaire was conducted anonymously, which was also informed to the participants, to ensure honesty and the accuracy of results. The work was surveyed through the WeChat program-Questionnaire Star and then published the QR-Code for the questionnaire on some social media platforms including WeChat, QQ, Instagram, and Weibo. Participants were asked to scan the code and complete the related multiple-choice questions.

\subsection{Measurement}

In the questionnaire, participants report age, gender, and choice between help-seeking and self-regulation as a way of coping with stress and pressure (See Fig 1). As for their ages, fifteen to eighteen is one age group, eighteen to fifty is another since eighteen was the full legal age in China. The gender options are male and female only. And from this anonymous questionnaire, we branched them down. The participants, who chose help-seeking ("help-seeking" group), were asked about the emotion that they experienced the most during the process, including shame, indifference, afraid, pride, and others (See Fig 2). The participants, who selected self-regulation ("self-regulation" group), had to answer why they chose self-regulation instead of help-seeking. The answer is made of 6 choices, covering financial concerns, shame-making, self-reliance, distrust of others, nonconfidence in the concept o psychiatry, and others (See Fig.3). The final question asked all participants about their attitudes towards people who seek help. There are 4 choices in this question, including indifference, envy, invalid, and others (See Fig.4).

The questionnaire was provided in both English and Chinese. And all individual results are promised confidentiality to prevent any interference or social pressure and thus to ensure the accuracy and reliability of our results.

\subsection{Participants}

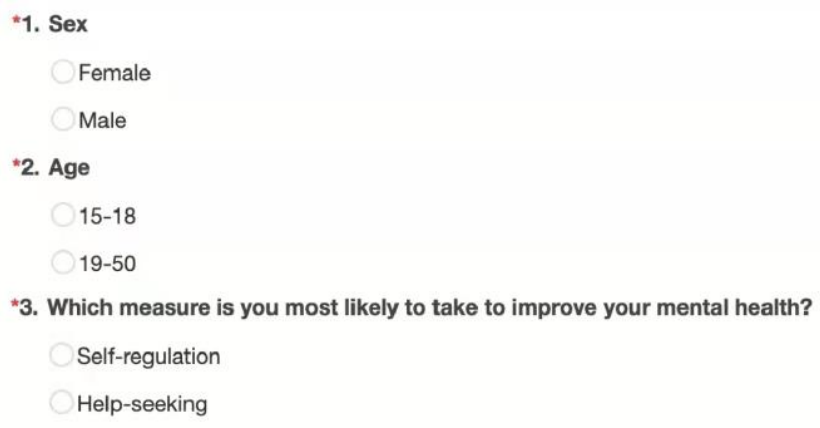

(Figure 1. gender, age, and coping choice) 


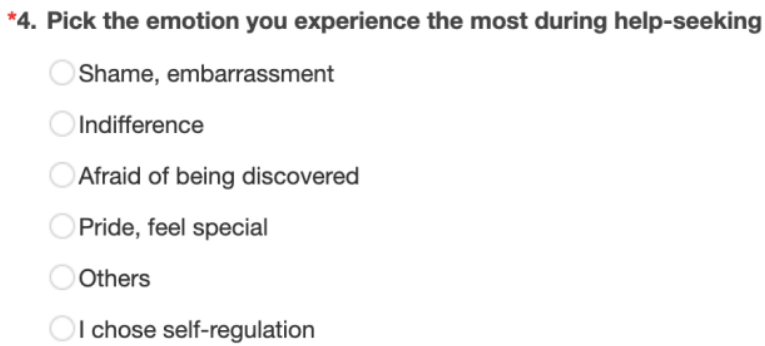

(Figure 2. the emotion of "help-seeking" group)

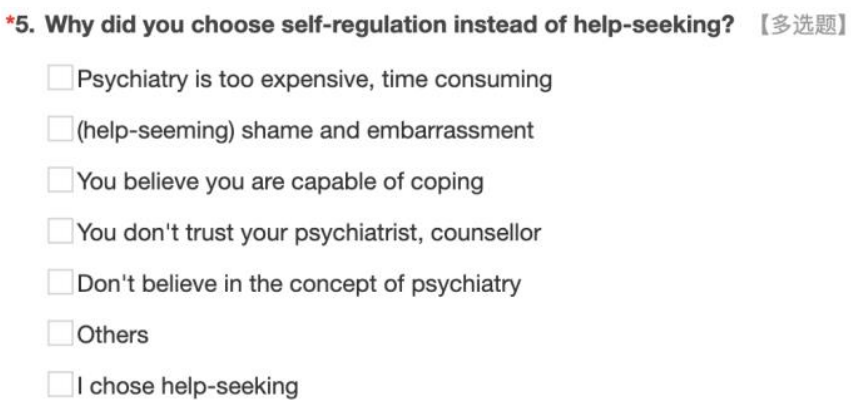

(Figure 3. the reason about not choosing help-seeking of "self-regulation" group)

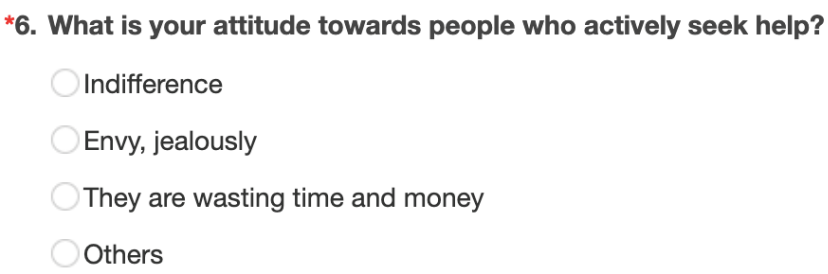

(Figure 4. attitudes of all participants)

In the first assumption, the work used 2 variables that are gender, whether they are male or female, and their coping strategy when they encountered psychological barriers, seeking help, or self-regulation.

In the second assumption, our purpose was to find out the association between gender and people's attitudes towards help-seeking. This assumption not only did the work take gender, age, and coping strategy into account but also their attitudes towards help-seeking and emotions that they experienced the most during seeking help. The work then proceeds to encode their coping choice and emotion in 3 choices, as either positive, neutral, or negative. The final variable is about all participants' attitudes towards help-seeking. Like the previous question, all of their subjective feelings were categorized as either positive, neutral, or negative.

\section{RESULT}

\subsection{Data analysis}

In this study, data collected from the questionnaire were analyzed. For statistical analyses, the work utilized Excel. The Chi-Square Test of Independence was used to determine whether or not there is a significant association between two categorical variables that are gender and coping method and whether or not there is a significant association between gender and their feelings of self-seeking.

\subsection{Assumption 1}

From the questionnaire, the result concluded that $64.7 \%$ of men chose self-regulation, and $55.3 \%$ of women chose self-regulation (See Figure 5).

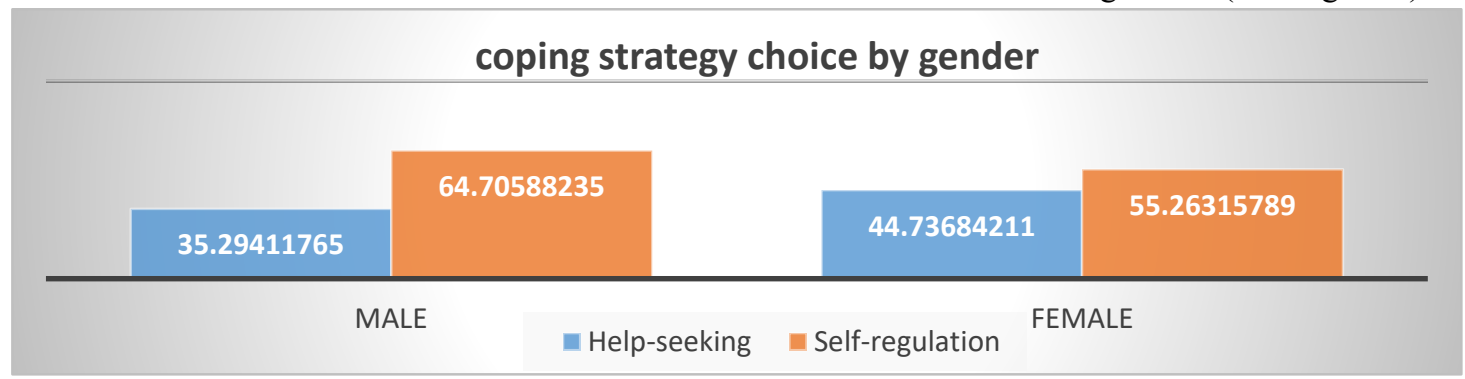

(Figure 5. coping strategy choice by gender) 
Based on assumption 1, the null hypothesis and alternative hypothesis were developed. Our question is that does coping strategy choice depends on gender. This work identified a null hypothesis as our coping strategy choice and gender are independent, and the alternative one is that men are significantly less likely to seek help than women.

The data of coping strategy selection of different genders from the questionnaire were extracted as the observed value of the first part (See Tab.1). Then using the formula calculate the Expected Value for each cell in the contingency table (See Tab.2).

The test statistic $\mathrm{X}^{\wedge} 2$ was calculated to investigate statistically (See Tab.3). The test statistic X2 turns out to be 1.125427483 and the corresponding $p$-value is 0.28875277 (See Tab.4). Significance was set at $\mathrm{a}=$ 0.05 . However, according to our data, since this $\mathrm{p}$-value is not less than 0.05 , the work fails to reject the null hypothesis. It means we can not accept the alternative hypothesis that men are significantly less likely to seek help than women.

(Table 1. the actual value of coping strategy of selection)

\begin{tabular}{|l|l|l|l|}
\hline & Help-seeking & Self-regulation & total \\
\hline male & 18 & 33 & 51 \\
\hline female & 34 & 42 & 76 \\
\hline total & 52 & 75 & 127 \\
\hline
\end{tabular}

(Table 2. the expected value of coping strategy of selection)

\begin{tabular}{|l|l|l|l|}
\hline & HS & SR & total \\
\hline male & 20.88188976 & 30.11811024 & 51 \\
\hline female & 31.11811024 & 44.88188976 & 76 \\
\hline total & 52 & 75 & 127 \\
\hline
\end{tabular}

(Table 3. the test statistic $\mathrm{X}^{\wedge} 2$ of coping strategy of selection)

\begin{tabular}{|l|l|l|l|}
\hline & HS & SR & total \\
\hline male & 0.397726868 & 0.275757295 & 51 \\
\hline female & 0.266895661 & 0.185047659 & 76 \\
\hline total & 52 & 75 & 127 \\
\hline
\end{tabular}

(Table 4. the process of assumption 1)

\begin{tabular}{|l|l|l|}
\hline$X^{\wedge}$ & 1.125427483 & SUM(N4:O5) \\
\hline deg_freedom & 1 & $(2-1)^{\wedge} 2=1$ \\
\hline$p$-value & 0.288752770 & CHISQ.DIST.RT(S3,S4) \\
\hline
\end{tabular}

\subsection{Assumption 2}

or neutral attitudes towards help-seeking and $86.8 \%$ of women had negative or neutral attitudes towards

From the questionnaire, $94.1 \%$ of men had negative help-seeking (See Figure 6).

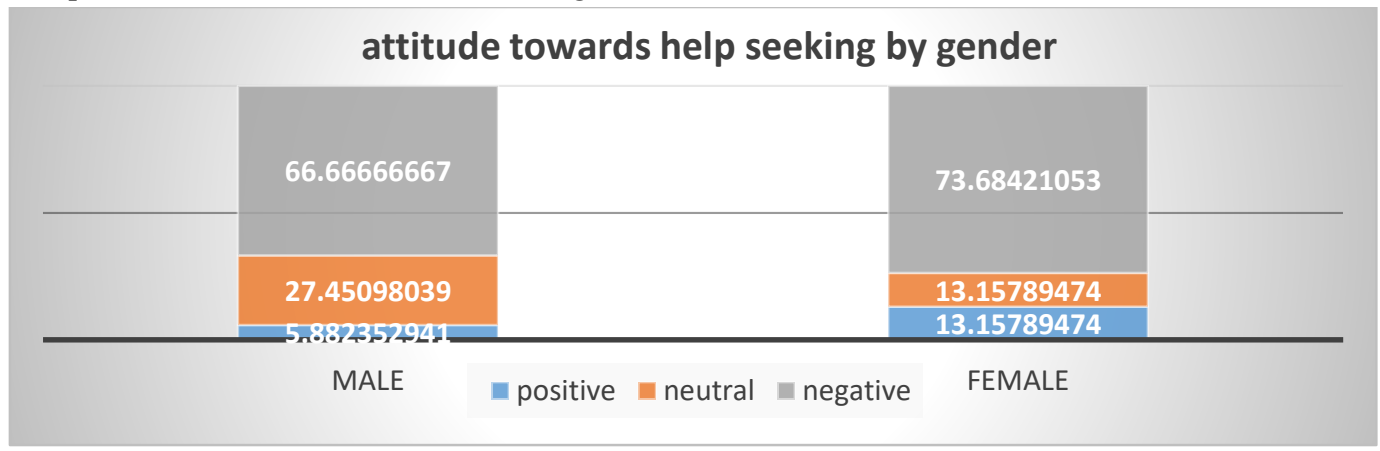

(Figure 6. attitude towards hep seeking by gender)

The purpose of the second work was to see if men are more resistant to asking for help. We carry it out basically in the same way as the first work. The null hypothesis is that Gender and help-seeking attitudes are independent. The alternative one is that men tend to hold more repellent attitudes towards help-seeking. 
After fetching the specific data from the questionnaire (See Tab.5), calculating the observed value (See Tab.6) and the test statistic chi-square (See Tab.7), the result could figure out that the p-value is 0.78487206 (See Tab.8). Significance was set at $\mathrm{a}=$
0.05 , since this p-value is also not less than 0.05 , the work fails to reject the null hypothesis. It means that we do not have sufficient evidence to say that there is an association between gender and an individual's help-seeking attitudes.

(Table 5. the actual value of individual's help-seeking attitudes)

\begin{tabular}{|c|c|c|c|c|}
\hline & positive & neutral & negative & total \\
\hline male & 3 & 14 & 34 & 51 \\
\hline female & 10 & 10 & 56 & 76 \\
\hline total & 13 & 24 & 90 & 127 \\
\hline
\end{tabular}

(Table 6. the expected value of individual's help-seeking attitudes)

\begin{tabular}{|c|c|c|c|c|}
\hline & positive & neutral & negative & total \\
\hline male & 5.220472441 & 9.637795276 & 36.14173228 & 51 \\
\hline female & 7.779527559 & 14.36220472 & 53.85826772 & 76 \\
\hline total & 13 & 24 & 90 & 127 \\
\hline
\end{tabular}

(Table 7. the test statistic $\mathrm{X}^{\wedge} 2$ of individual's help-seeking attitudes)

\begin{tabular}{|c|c|c|c|c|}
\hline & positive & neutral & negative & total \\
\hline male & 0.944454341 & 1.974396583 & 0.126917469 & 51 \\
\hline female & 0.633778571 & 1.324924023 & 0.085168301 & 76 \\
\hline total & 13 & 24 & 90 & 127 \\
\hline
\end{tabular}

(Table 8. the process of assumption 2)

\begin{tabular}{|c|c|c|}
\hline$X^{\wedge}$ & 5.089639288 & SUM(P11:R12) \\
\hline deg_freedom & 2 & $(3-1)^{\star}(2-1)=2$ \\
\hline$p$-value & 0.078487206 & CHISQ.DIST.RT $(v 10, v 11)$ \\
\hline
\end{tabular}

\section{CONCLUSION}

The purposes of this study were to explore the association between gender and coping strategies of psychological disorders, and between gender and peoples' attitudes towards help-seeking. Results demonstrate that these are not necessarily true. Because in each assumption, the p-value is not less than 0.05 , we cannot conclude that these two associations are accurate.

There are several limits to this approach. Firstly, regarding the limitations of age and generational differences, it can be argued that it is difficult to interpret such results in the context of the absence of a large number of participants. And the sample size in this study was not considered large enough to represent the whole population. This study cannot deny the presence of some sample selection biases because of the unevenly distributed sample on age, and all of it about 77.2 (rounded to three significant figures) percent of our participants are from 15 18.

Also, current evidence relies on participants mainly from Chengdu and Shanghai. These cities tend to be more open and accepting. Thus, people may have more encouraged to seek help with fewer barriers, such as access to professionals, to medications, and they are also more likely to be economically and time wisely available to seek help. Furthermore, in 2019, the Education Bureau of the People's Republic of China issued a plan to provide mental health services for students in all schools by the end of 2022. People tend to be more educated on the subject of mental health, and they tend to be more psychologically open. Therefore, we think this shows that our results are only applicable to certain backgrounds.

Thirdly, many factors influence the choices and attitudes of teenagers (the age range from 15 to 18.), including self-awareness and financial concerns, and some factors may particularly affect older persons, covering misunderstandings about psychological counseling. According to some researchers, middle-aged people seek help most often, followed by older people and younger people least.[8] Thus, these external factors may also influence people's current coping strategies when they encountered mental disorders and their attitudes towards seeking help. 
Here the work compares the results of the proposed method with those of the traditional methods. They have demonstrated that men are far less likely than women to seek help for mental health.[6] Besides, social, self-stigma, and gender role conflict experience were significantly correlated with males' attitudes towards psychological help, and played a predictive role.[12]

Contrary to the findings above, the work did not find a significant association, partly because of the limitations. Nevertheless, those results from our research still provide a potential mechanism for studying gender and help-seeking. Our data suggest that we still have a long way to go. Future research should consider the potential effects of those limitations more carefully. The possibility of the association between gender and coping strategies, and between gender and attitudes towards help-seeking warrants further investigation.

\section{REFERENCES}

[1] Ford, D. E., \& Erlinger, T. P. (2004). Depression and C-reactive protein in US adults: data from the Third National Health and Nutrition Examination Survey. Archives of internal medicine, 164(9), 1010-1014 https://doi.org/10.1001/archinte.164.9.1010

[2] Oquendo, M. A., Ellis, S. P., Greenwald, S., Malone, K. M., Weissman, M. M., Mann, J. J. (2001). Ethic and sex differences in suicide rates relative to major depression in the United States. American Journal of Psychiatry, 158, 1652-1658.

[3] McManus S, Bebbington P, Jenkins R, Brugha T. (eds.) (2016) Mental health and wellbeing in England: Adult Psychiatric Morbidity Survey 2014. Leeds: NHS Digital.

[4] Pederson, E. L., \& Vogel, D. L. (2007). Male gender role conflict and willingness to seek counseling: Testing a mediation model on college-aged men. Journal of Counseling Psychology, 54(4), 373-384.

[5] Möller-Leimkühler, A. (2002). Barriers to help-seeking by men: A review of sociocultural and clinical literature with particular reference to depression. Journal of Affective Disorders, 71(1), 1-9. doi:10.1016/S0165-0327(01)00379-2

[6] Addis, M. E., \& Mahalik, J. R. (2003). Men, masculinity, and the contexts of help seeking. The American psychologist, 58(1), 5-14. https://doi.org/10.1037/0003-066x.58.1.5

[7] Wang, Y., Hunt, K., Nazareth, I., Freemantle, N., \& Petersen, I. (2013). Do men consult less than women? An analysis of routinely collected UK general practice data. BMJ Open, 3(8), e003320. doi: 10.1136/bmjopen-2013-003320

[8] Kessler, R., Demler, O., Frank, R., Olfson, M., Pincus, H., \& Walters, E. et al. (2005). Prevalence and Treatment of Mental Disorders, 1990 to 2003. New England Journal Of Medicine, 352(24), 2515-2523. doi: 10.1056/nejmsa043266

[9] Wang, P., Aguilar-Gaxiola, S., Alonso, J., Angermeyer, M., Borges, G., \& Bromet, E. et al. (2007). Use of mental health services for anxiety, mood, and substance disorders in 17 countries in the WHO world mental health surveys. The Lancet, 370(9590), 841-850. doi: 10.1016/s0140-6736(07)61414-7

[10] Mackenzie, C. S., Scott, T., Mather, A., \& Sareen, J. (2008). Older adults' help-seeking attitudes and treatment beliefs concerning mental health problems. The American journal of geriatric psychiatry : official journal of the American Association for Geriatric Psychiatry, 16(12), 1010 1019.

https://doi.org/10.1097/JGP.0b013e31818cd3be

[11] Wang, P. S., Aguilar-Gaxiola, S., Alonso, J., Angermeyer, M. C., Borges, G., Bromet, E. J., Bruffaerts, R., de Girolamo, G., de Graaf, R., Gureje, O., Haro, J. M., Karam, E. G., Kessler, R. C., Kovess, V., Lane, M. C., Lee, S., Levinson, D., Ono, Y., Petukhova, M., Posada-Villa, J., ... Wells, J. E. (2007). Use of mental health services for anxiety, mood, and substance disorders in 17 countries in the WHO world mental health surveys. Lancet (London, England), 370(9590), 841-850. https://doi.org/10.1016/S0140-6736(07)61414-7

[12] Wahto, R., \& Swift, J. (2014). Labels, Gender-Role Conflict, Stigma, and Attitudes Toward Seeking Psychological Help in Men. American Journal Of Men's Health, 10(3), 181-191. doi: $10.1177 / 1557988314561491$ 